Eric Leichtnam $\cdot$ Xiang Tang $\cdot$ Alan Weinstein

\title{
Poisson geometry and deformation quantization near a strictly pseudoconvex boundary
}

Received April 24, 2006

\begin{abstract}
Let $X$ be a complex manifold with strongly pseudoconvex boundary $M$. If $\psi$ is a defining function for $M$, then $-\log \psi$ is plurisubharmonic on a neighborhood of $M$ in $X$, and the (real) 2-form $\sigma=i \partial \bar{\partial}(-\log \psi)$ is a symplectic structure on the complement of $M$ in a neighborhood of $M$ in $X$; it blows up along $M$.

The Poisson structure obtained by inverting $\sigma$ extends smoothly across $M$ and determines a contact structure on $M$ which is the same as the one induced by the complex structure. When $M$ is compact, the Poisson structure near $M$ is completely determined up to isomorphism by the contact structure on $M$. In addition, when $-\log \psi$ is plurisubharmonic throughout $X$, and $X$ is compact, bidifferential operators constructed by Engliš for the Berezin-Toeplitz deformation quantization of $X$ are smooth up to the boundary. The proofs use a complex Lie algebroid determined by the CR structure on $M$, along with some ideas of Epstein, Melrose, and Mendoza concerning manifolds with contact boundary.
\end{abstract}

Keywords. Poisson structure, pseudoconvexity, plurisubharmonic function, contact structure, Lie algebroid

\section{Introduction}

Let $u$ be a real-valued function on a complex manifold $X$. The 2-form $\sigma=\sigma_{u}=i \partial \bar{\partial} u$ is of type $(1,1)$, real, and exact (since $\partial \bar{\partial}=d \bar{\partial})$. In addition, $\sigma(x, y)=\sigma(J x, J y)$, where $J$ is the complex structure viewed as an endomorphism of $T X$, so the "hermitian hessian" bilinear form $g_{u}(x, y)=\sigma(x, J y)$ is symmetric. When $g_{u}$ is positive definite, $u$ is said to be strongly plurisubharmonic. In this case, $g_{u}$ is a Kähler metric on $X$, and the form $\sigma$ is nondegenerate, i.e. symplectic. The function $u$ is called a Kähler potential for $g_{u}$. Conversely, the Dolbeault lemma implies that any Kähler metric on $X$ arises from a potential on a neighborhood of each point of $X$. While the local geometry of the Kähler metric depends very much on the choice of potential, the symplectic form has no local

E. Leichtnam: CNRS and Institut Mathématique de Jussieu, Etage 7 E, 175 Rue du Chevaleret, 75013 Paris, France

X. Tang: Department of Mathematics, Washington University, St. Louis, MO 63130, USA; e-mail: xtang@math.wustl.edu

A. Weinstein: Department of Mathematics, University of California, Berkeley, CA 94720, USA; e-mail: alanw@math.berkeley.edu

Mathematics Subject Classification (2000): Primary 32T15; Secondary 53D10, 53D17 
invariants. (See [7] for global results about the symplectic geometry of Kähler manifolds with global potential functions.)

In this paper, we will investigate what happens when the complex manifold $X$ has a boundary $M$. The hyperplane field $F_{M, X}=T M \cap J T M$ is called the maximal complex subbundle of $T M$. A defining function for $M$ is a smooth nonnegative function $\psi$ whose zero set is $M$, and which has no critical points on $M$. The restriction to $F_{M, X}$ of the hermitian hessian $g_{\psi}$ is, up to a positive conformal factor, independent of the choice of defining function. The associated invariant object, a symmetric bilinear form with values in the conormal bundle of $M$, is called the Levi form of $M . M$ is called Levi nondegenerate when this form is nondegenerate, and strongly pseudoconvex when it is negative definite. $M$ is Levi nondegenerate if and only if $F_{M, X}$ is a contact structure.

When $M$ is strongly pseudoconvex, $-\log \psi$ is strongly plurisubharmonic on $U \backslash M$ for some neighborhood $U$ of $M$ in $X$, and $\sigma_{-\log \psi}$ on $U \backslash M$ is a symplectic structure which blows up along $M$. We will show that the corresponding Poisson structure $\pi_{-\log \psi}$ extends smoothly to $M$, along which it is zero.

In fact, using Epstein, Melrose and Mendoza's [11] notion of $\Theta$-structure, we will show that, whenever $M$ is Levi nondegenerate, the local isomorphism type of the Poisson structure $\pi_{-\log \psi}$ is independent of everything but the dimension of $X$. The local model is LeBrun's [22] Poisson structure on the normal bundle to a contact structure, and equivalence with this model gives the smoothness of $\pi_{-} \log \psi$ up to the boundary.

Contact structures also play a role in the global (on $M$ ) version of this result: the germ along $M$ of the Poisson structure is determined, up to diffeomorphisms fixing $M$, by the contact structure $F_{M, X}$. Its isomorphism class is thus independent of the choice of the defining function and of the choice of (compatible) complex structure. Again, the model for $\pi_{-\log \psi}$ is given by LeBrun's construction.

Similar results, for flows and infinitesimal deformations on pseudoconvex manifolds, have been obtained by Korányi and Reimann [21], [29].

We turn next to quantization. When $X$ is compact and $\psi$ is strongly plurisubharmonic throughout $X, \pi_{-\log \psi}$ is the semiclassical commutator of the Berezin-Toeplitz deformation quantization product on $X$. The construction of this product involves the action of smooth functions on $X$ by multiplication and projection on a parameterized family of weighted Bergman spaces of holomorphic functions on the interior of $X$. The BerezinToeplitz product was analyzed in the pseudoconvex setting by Engliš [8], following many earlier studies on closed manifolds. He showed that the induced product on smooth functions has an asymptotic expansion in the weight parameter; the terms in the expansion are bidifferential operators whose coefficients are algebraic combinations of the Kähler metric, its curvature, and covariant derivatives thereof.

We will use the notion of complex Lie algebroid to show that all the bidifferential operators in the Berezin-Toeplitz-Engliš quantization are smooth up to the boundary. In fact, we will show something stronger. Karabegov [19] has defined a notion of quantization with separation of variables on a Kähler manifold, and it is known that the Berezin-Engliš-Toeplitz quantization has this property on the interior of $X$. We will extend Karabegov's definition by introducing a notion of para-Kähler structure on a com- 
plex Lie algebroid, and we will show that the separation of variables property then holds up to the boundary.

In the future, we plan to extend our results to more general manifolds, replacing the global strongly plurisubharmonic function $\psi$ by a family of local functions obtained from a connection on a hermitian line bundle. These results will be used in the proof of a topological formula for the relative index of CR structures defined by Epstein [9] which was conjectured by Atiyah and Weinstein [31]. (A proof of the conjecture by Epstein [10] has recently appeared, but his methods are quite different from ours.)

\section{Normal forms}

For most of this section, we will forget about complex geometry and look at what Epstein, Melrose, and Mendoza [11] call $\Theta$-structures. $M$ will now be the boundary of any manifold $X$ of real dimension $2 n+2$.

Definition 2.1. An EMM form is a 1-form $\Theta$ on $X$ whose pullback to $M$ is a contact form.

Our model example of an EMM form will be the pullback to $X=M \times \mathbb{R}^{+}$of a contact form $\phi$ on $M$; we identify $M$ with the zero set of the coordinate function $r$ on $\mathbb{R}^{+}=$ $[0, \infty)$. The symplectic form $d(\Theta / r)$ blows up along $M$, but LeBrun [22] observed that the Poisson structure inverse to $d(\Theta / r)$ extends to a smooth Poisson structure on $M \times \mathbb{R}^{+}$. If $\phi=d u+\sum p_{j} d q^{j}$ in local coordinates $(u, q, p)$ on $M$, then, on $M \times \mathbb{R}^{+}$,

$$
d(\Theta / r)=\left(1 / r^{2}\right)\left[-d r \wedge\left(d u+\sum p_{j} d q^{j}\right)+r d p_{j} \wedge d q^{j}\right],
$$

and the Poisson structure corresponding 11 to $-d(\Theta / r)$ is

$$
\Pi=r\left[\left(r \frac{\partial}{\partial r}+\sum p_{j} \frac{\partial}{\partial p_{j}}\right) \wedge \frac{\partial}{\partial u}+\sum \frac{\partial}{\partial q^{j}} \wedge \frac{\partial}{\partial p_{j}}\right] .
$$

\subsection{Local normal form}

We will use the following local theorem in order to obtain a global normal form. (It would be nice to get the global form all at once, but we do not know how to do it.)

Theorem 2.2. Let $\Theta$ be an EMM form on the manifold $X$ with boundary $M$, and let $\psi$ be a defining function for the boundary. Then, near each $m \in M$, there exist local coordinates $(q, p, u, r)$ on $X$ in which $\psi=r$ and $d(\Theta / \psi)$ has the form (1) on the complement of $M$. In particular, $-d(\Theta / \psi)$ is symplectic on the complement of $M$ in a neighborhood of $m$ in $X$; the corresponding Poisson structure on this neighborhood has the local normal form (2).

\footnotetext{
1 There is a choice of sign when one says that a Poisson structure corresponds to a symplectic structure. Unlike LeBrun [22], we use the convention in which $d q \wedge d p$ corresponds to the relation $\{q, p\}=1$.
} 
Proof. We begin by setting $r=\psi$. By the Darboux theorem for contact 1-forms [4], we may find local coordinates on $M$ for which the pullback of $\Theta$ has the expression $d u+$ $p_{j} d q^{j}$. In fact, the pullback of $\Theta$ to each level of $r$ near $M$ is still a contact structure, so we may choose coordinates on all these levels, depending smoothly on $r$, so that the pullbacks all have the same form. It follows that $\Theta$ itself may be written as $d u+p_{j} d q^{j}+a d r$, where $a$ is a smooth function of all the variables.

To eliminate the term $a d r$, we use Moser's method, i.e. constructing a diffeomorphism (preserving $r$ and fixed on $M$ ) by integrating a time-dependent vector field $X_{t}$. As usual, we define $\Theta_{t}$ by interpolation as $d u+p_{j} d q^{j}+t a d r$ and choose $X_{t}$ to satisfy the condition $\left.X_{t}\right\lrcorner d\left(\Theta_{t} / r\right)=-(a / r) d r$. Now

$$
\begin{aligned}
d\left(\Theta_{t} / r\right) & =(1 / r) d \Theta_{t}-\left(1 / r^{2}\right) d r \wedge \Theta_{t} \\
& =(1 / r)\left(d p_{j} \wedge d q^{j}+t d a \wedge d r\right)-\left(1 / r^{2}\right) d r \wedge\left(d u+p_{j} d q^{j}+t a d r\right) .
\end{aligned}
$$

If we take $X_{t}$ to be a function $f_{t}(q, p, u, r)$ times the (Reeb) vector field $\partial / \partial u$, it will be tangent to the levels of $r . X_{t}$ must satisfy the equation

$$
X_{t} \downarrow d\left(\Theta_{t} / r\right)=(t / r)\left(f_{t}(\partial a / \partial u)+(1 / r) f_{t}\right) d r=-(a / r) d r
$$

which has the solution $f_{t}=-r a /(1+r t \partial a / \partial u)$. The denominator is invertible near $M$, and the factor of $r$ in the numerator of $f_{t}$ insures that $X_{t}$ vanishes along $M$, in addition to being smooth and tangent to the levels of $r$.

\subsection{Global normal form}

To put $\Theta$ in normal form on a neighborhood of the entire boundary, we can no longer fix the $\psi$ levels, because the characteristic line element field of the pullback of $d(\Theta / \psi)$ has a global dynamics which may vary from one $\psi$ level to another. This also makes it impossible to use the Darboux theorem as we did for the local normal form. Instead, we use Gray's theorem, which asserts that deformations of a contact structure on a compact manifold are trivial. We refer the reader to Cannas da Silva [4] for a proof, noting for use below that the transformations in Gray's theorem may easily be chosen to depend smoothly on a parameter.

Like the local model, the global normal form comes from LeBrun [22]. For any contact structure $F \subset T M$, the conormal bundle $v^{*}=(T M / F)^{*}$ may be identified with the 1-dimensional subbundle of $T^{*} M$ consisting of all real multiples of any contact form defining the contact structure. The pullback to $v^{*}$ of the canonical symplectic structure on $T^{*} M$ is nondegenerate on the complement of the zero section of $v^{*}$. We may identify this complement by "inversion" with the complement of the zero section in the normal bundle $v=T M / F$. LeBrun shows that the Poisson structure corresponding to this form on $v$ now extends smoothly over the zero section. When $v$ is oriented, a choice of contact form identifies $v$ with $M \times \mathbb{R}$, and the nonnegative normal bundle $v^{+}$is identified with $M \times \mathbb{R}^{+}$. The Poisson structure is given by (2). 
Theorem 2.3. Let $\Theta$ be an EMM form for the manifold $X$ with boundary $M$, and $\psi a$ defining function for the boundary. Then a neighborhood of $M$ in $X$, with the structure corresponding to $-d(\Theta / \psi)$, is Poisson isomorphic to a neighborhood of the zero section in the nonnegative normal bundle $v^{+}$, with the LeBrun-Poisson structure associated to the contact structure induced by $\theta$ on $M$.

Proof. As in the proof of Theorem 2.2 we will work with the 2-forms. Since the diffeomorphism we construct will be smooth along the singular locus of these forms, it will automatically be a Poisson isomorphism.

To begin, we identify both $X$ near $M$ and $v^{+}$near the zero section with a neighborhood of the zero section in the trivial bundle $M \times \mathbb{R}^{+}$. For $v^{+}$, we use the trivialization of $v$ given by the contact form which is the pullback of $\Theta$ to $M$. For $X$ near $M$, we first arrange that the projection onto $\mathbb{R}$ is the given function $\psi$, i.e. we set $r=\psi$; we then use Gray's theorem to arrange that the projection of each $\psi$ level onto $M$ is a contact diffeomorphism.

Let us write $\Theta_{0}$ for the standard form $d u+\sum p_{j} d q^{j}$ (independent of $r$ ) and $\Theta_{1}$ for the given form. Since $\Theta_{1}$ defines the same contact structure as $\Theta_{0}$ on each level of $r$, and it agrees with $\Theta_{0}$ on the zero level, $\Theta_{1}=\Theta_{0}+a d r+b r \Theta_{0}$, where $a$ and $b$ are smooth functions. As before, we linearly interpolate to get $\Theta_{t}=\Theta_{0}+t a d r+t b r \Theta_{0}$. Note for later use in this proof that these are all EMM forms, so we can apply Theorem 2.2 to put them in local normal form.

Once again, we seek a time-dependent vector field $X_{t}$ to generate our normalizing transformation. The required condition on this vector field is

$$
X_{t}-d\left(\Theta_{t} / r\right)=-(a / r) d r+b \Theta_{0} .
$$

The unique solution of this equation is (on the complement of $M$ ) the contraction of the right hand side with the Poisson structure corresponding to $-d\left(\Theta_{t} / r\right)$. We already know from the local normal form that this Poisson structure vanishes along $M$, so the contraction extends smoothly over $M$. It remains to show that the contraction vanishes along $M$. For this, it suffices to show that the contraction with $d r$ vanishes to second order. But, from the local normal form (2), we find immediately that, in normal form coordinates, this contraction is equal to $r^{2} d u$, and our proof is complete.

Remark 2.4. Although the normal form theorem above may suggest that the LeBrunPoisson structure is rigid with respect to arbitrary higher-order perturbations, this is in fact not the case. For instance, when $M$ is 1-dimensional, the Poisson structure is simply $\Pi=r^{2} \partial / \partial r \wedge \partial / \partial u$. This structure is exact in the sense that there is a vector field $\xi$ (namely $\partial / \partial r$ ) satisfying $[\xi, \Pi]=\Pi$, but the Poisson structure $\left(r^{2}+r^{3}\right) \partial / \partial r \wedge \partial / \partial u$ does not admit such a $\xi$ if $M$ is a circle. A related fact is that $\left(1 /\left(r^{2}+r^{3}\right)\right) d r \wedge d u$ is not $d(\Theta / r)$ for any EMM form $\Theta$.

We also note the following relative form of Theorem 2.3

Corollary 2.5. If $\Theta_{0}$ and $\Theta_{1}$ are EMM forms which agree to infinite order along $M$, then there is a diffeomorphism germ on $X$ along $M$ which agrees with the identity to infinite order along $M$ and pulls back $\Theta_{1}$ to $\Theta_{0}$. 
Proof. By Theorem 2.3, we may assume that $X=M \times[0,1)$ and that $\Theta_{0}$ is the standard form. We then repeat the proof of Theorem 2.3, the functions $a$ and $b$ vanish to infinite order along $M$, hence so does the vector field $X_{t}$ which generates the normalizing transformation.

\subsection{Application to pseudoconvex boundaries}

Let $X$ be a complex manifold with boundary $M$. By elementary calculus on complex manifolds,

$$
\bar{\partial} f=\frac{1}{2}\left(d f+i J^{*} d f\right)
$$

for any smooth function $f$, where $J: T X \rightarrow T X$ is the almost complex structure. It follows that

$$
i \partial \bar{\partial}(-\log \psi)=i d \bar{\partial}(-\log \psi)=\frac{1}{2} d\left(J^{*} \frac{d \psi}{\psi}\right)=d\left(\frac{1}{2} J^{*} d \psi / \psi\right) .
$$

Let $\psi$ be a defining function for the boundary and set $r=\psi$ and $\Theta=\frac{1}{2} J^{*} d \psi$. We will show that $\Theta$ is an EMM form. In fact, in $T_{M} X, T M$ is $\operatorname{ker} d \psi$, so

$$
\operatorname{ker} \Theta \cap T M=\operatorname{ker} J^{*} d \psi \cap T M=J(\operatorname{ker} d \psi) \cap T M=J T M \cap T M=F_{M, X},
$$

the maximal complex subbundle of $T M$. Since $M$ is Levi nondegenerate, $F_{M, X}$ is a contact structure, and hence $\Theta$ is an EMM form. It follows that all the results of this section apply to the form $\sigma_{-\log \psi}$ and the corresponding Poisson structure $\pi_{-\log \psi}$.

We remark that our results correspond very closely to results on flows and deformations due to Korányi and Reimann [21], [29]. Since the Poisson structure determines the contact structure on the boundary, our methods also give a simple proof of their (easier) converse result that a smooth map which is symplectic on the interior must be contact on the boundary.

Here is a direct nondegeneracy proof which is independent of the normal form theorem. It involves a volume element computation which we will use in the proof of Proposition 4.7 below.

Proposition 2.6. Let $\psi$ be a defining function for the boundary $M$ of $X$. The closed 2form $\sigma_{-\log \psi}$ is nondegenerate on a neighborhood of $M$ in the interior of $X$ if $M$ is Levi nondegenerate.

Proof. We compute:

$$
(1 / i) \sigma_{-\log \psi}=\partial \bar{\partial}(-\log \psi)=-\partial\left(\frac{\bar{\partial} \psi}{\psi}\right)=\frac{-\psi \partial \bar{\partial} \psi+\partial \psi \wedge \bar{\partial} \psi}{\psi^{2}} .
$$

Raising this 2 -form to the $(n+1)$ st power gives

$$
\left(-\frac{\partial \bar{\partial} \psi}{\psi}+\frac{\partial \psi \wedge \bar{\partial} \psi}{\psi^{2}}\right)^{n+1}=\left(-\frac{\partial \bar{\partial} \psi}{\psi}\right)^{n+1}+\left(-\frac{\partial \bar{\partial} \psi}{\psi}\right)^{n} \wedge \frac{\partial \psi \wedge \bar{\partial} \psi}{\psi^{2}}
$$


which is $\psi^{-(n+2)}$ times

$$
\psi(-\partial \bar{\partial} \psi)^{n+1}+(-\partial \bar{\partial} \psi)^{n} \wedge \partial \psi \wedge \bar{\partial} \psi
$$

Our lemma will be proven if we can show that this form is nonzero near $M$ when $M$ is Levi nondegenerate. Since the first term vanishes along $M$ it suffices to show that the second is nonzero. Since $\partial \psi \wedge \bar{\partial} \psi$ is nonzero and annihilates the contact structure $F_{M, X}$, the nonvanishing of the term is equivalent to nondegeneracy of the restriction to $F_{M, X}$ of $(-\partial \bar{\partial} \psi)^{n}$. But $-\partial \bar{\partial} \psi$ is just the 2 -form associated via $J$ to the Levi form.

Remark 2.7. There are natural 1-1 correspondences among several bundles along the boundary $M$ whose sections admit natural simple and transitive actions of the smooth positive functions on $M$ :

1. 1-jets along $M$ of defining functions.

2. Sections of the conormal bundle $T M^{\perp} \subset T_{M}^{*} X$ which are "positive" in the sense that they take positive values on inward-pointing vectors in $T_{M} X$.

3. Contact forms realizing the cooriented contact structure on $M$.

4. Volume elements on $M$ compatible with the natural boundary orientation of $M$.

The correspondence $1 \leftrightarrow 2$ is almost tautological, since any section of the conormal bundle may be realized as the derivative along $M$ of a defining function. (For instance, if we multiply the defining function $\psi$ by a positive function $\lambda$, its differential along $M$ is also multiplied by $\lambda$.) For $2 \leftrightarrow 3$, we associate to each positive section $\alpha$ of the conormal bundle the pullback to $M$ of $J^{*} \alpha$. (To go in the other direction, we extend any contact form along $M$ to a section of $T_{M}^{*} X$ by requiring it to annihilate $J T M$.) Finally, for $3 \leftrightarrow 4$, we associate to each contact form $\theta$ the volume element $\theta \wedge(d \theta)^{n}$. Rescaling $\theta$ by $\lambda$ multiplies the volume element by $\lambda^{n+1}$.

\section{Geometry on complex Lie algebroids}

Complex Lie algebroids were defined in [5] and have been studied in more detail in [1] and [32]. In this section, we will review the definitions and use a complex Lie algebroid to "regularize" the geometry of a complex manifold near a pseudoconvex boundary.

\subsection{Definition and first examples}

We recall that a Lie algebroid over a smooth manifold $X$ is a real vector bundle $E$ over $X$ with a Lie algebra structure (over $\mathbb{R}$ ) on its sections and with a bundle map $\rho$ (called the anchor) from $E$ to the tangent bundle $T M$, satisfying the Leibniz rule

$$
[a, f b]=f[a, b]+(\rho(a) f) b
$$

for sections $a$ and $b$ and smooth functions $f$. 
There is an analogous definition for complex manifolds, in which $E$ is a holomorphic vector bundle over $X$, and the Lie algebra structure is defined on the sheaf of local sections. Such objects are called complex Lie algebroids by Chemla [6], but, as in [5], we will reserve this term for the "hybrid" concept defined below.

From now on, $C^{\infty}(X)$ will denote the algebra of smooth complex-valued functions on a manifold $X$.

Definition 3.1. A complex Lie algebroid over a smooth (real) manifold $X$ is a complex vector bundle $E$ over $X$ with a Lie algebra structure (over $\mathbb{C}$ ) on its space $\mathcal{E}$ of sections and a bundle map $\rho$ (called the anchor) from $E$ to the complexified tangent bundle $T_{\mathbb{C}} X$, satisfying the Leibniz rule

$$
[a, f b]=f[a, b]+(\rho(a) f) b
$$

for $a$ and $b$ in $\mathcal{E}$ and $f$ in $C^{\infty}(X)$.

A "trivial" class of complex Lie algebroids consists of the complexifications of real Lie algebroids, such as $T_{\mathbb{C}} X$ itself. More interesting are general "involutive systems," which are subbundles of $T_{\mathbb{C}} X$ whose spaces of sections are closed under the (complexified) bracket of vector fields. (Up to isomorphism, these are just the complex Lie algebroids with injective anchor.) Among these are the complex structures and CR structures. By a complex structure, we mean here a subbundle of the form $E=T_{J}^{0,1} X=\{v+i J v$ | $v \in T X$, where $J: T X \rightarrow T X$ is an integrable almost complex structure. These are characterized among all complex subbundles by closure under bracket and the algebraic property that $T_{\mathbb{C}} M=E \oplus \bar{E}$. By a CR structure, we mean an involutive system $E$ for which $E \cap \bar{E}=\{0\}$ and $E+\bar{E}$ has codimension 1 in $T_{\mathbb{C}} X$. Any real hypersurface $M$ in a complex manifold $X$ (such as a boundary) inherits a CR structure, namely the intersection $G_{M, X}=T_{\mathbb{C}} M \cap T_{J}^{0,1} X$. (The problem of realizing a given CR structure in this way has been crucial in the development of linear PDE theory.) The sum $G_{M, X} \oplus \bar{G}_{M, X}$ is the complexification of the maximal complex subbundle $F_{M, X}$.

The main example of our paper, introduced in Section 3.3, will not have an injective anchor. However, its anchor will be bijective on an open dense subset of the base manifold $X$, and the use of Lie algebroids with this property could be viewed as an application of the method of moving frames, extended to allow certain "singular" frame fields.

No discussion of complex Lie algebroids should fail to mention the important example of generalized complex structures [15], [18], but having thus fulfilled this obligation, we will not discuss them further.

\subsection{Some constructions on complex Lie algebroids}

Many notions can be extended from real to complex Lie algebroids without any extra effort. Here are some which we will use later. Parts of this section are almost transcribed verbatim from [26]. Note that all the constructions below are local and may thus be carried out on the sheaf level. 
Definition 3.2. Let $(E, \rho,[]$,$) be a complex Lie algebroid over X$. The $E$-de Rham complex $\left({ }^{E} \Omega^{\bullet}(X),{ }^{E} d\right)$ is given by ${ }^{E} \Omega^{\bullet}(X)=\Gamma\left(\bigwedge^{\bullet}\left(E^{*}\right)\right)$, with

$$
\begin{aligned}
{ }^{E} d \mu\left(a_{1}, \ldots, a_{k+1}\right)= & \sum_{i}(-1)^{i} \rho\left(a_{i}\right) \mu\left(a_{1}, \ldots, \hat{a}_{i}, \ldots, a_{k+1}\right) \\
& +\sum_{i<j}(-1)^{i+j-1} \mu\left(\left[a_{i}, a_{j}\right], a_{1}, \ldots, \hat{a}_{i}, \ldots, \hat{a}_{j}, \ldots, a_{k+1}\right) .
\end{aligned}
$$

Elements of the complex are called $E$-differential forms on $X$; the cohomology of ${ }^{E} d$ is denoted by ${ }^{E} H^{\bullet}(X)$ and is called the $E$-de Rham cohomology of $X$.

When $E=T_{\mathbb{C}} X,{ }^{E} H^{\bullet}(X)$ is the usual de Rham cohomology of $X$ with complex coefficients.

Definition 3.3. An E-connection on a vector bundle $F$ over $X$ is a map

$$
(a, \gamma) \mapsto \nabla_{a} \gamma
$$

from $\mathcal{E} \times \Gamma(F)$ to $\Gamma(F)$ which is $C^{\infty}(X)$-linear in a and satisfies the Leibniz rule

$$
\nabla_{a}(f \gamma)=f \nabla_{a} \gamma+(\rho(a) f) \gamma
$$

for $f \in C^{\infty}(X)$. Equivalently, an E-connection on $F$ is a map $\nabla: \Gamma(F) \rightarrow \Gamma\left(E^{*} \otimes F\right)$ satisfying $\nabla(f \gamma)=f \nabla \gamma+{ }^{E} d f \otimes \gamma$.

Like an ordinary linear connection, an $E$-connection extends to a map

$$
\nabla: \Gamma\left(\bigwedge^{\bullet}\left(E^{*}\right) \otimes F\right) \rightarrow \Gamma\left(\bigwedge^{\bullet+1}\left(E^{*}\right) \otimes F\right) .
$$

The square of this extended operator is given by $\nabla^{2} \gamma=R \wedge \gamma$, where the curvature $R$ is the element of $\bigwedge^{2}\left(E^{*}\right) \otimes \operatorname{End}(F)$ defined by

$$
R(a, b)=\nabla_{a} \nabla_{b}-\nabla_{b} \nabla_{a}-\nabla_{[a, b]} .
$$

When the curvature is zero, the connection is also called a representation of $E$ on $F$.

When $F=E$, we may also define the torsion of $\nabla$ by the formula $T(a, b)=\nabla_{a} b-$ $\nabla_{b} a-[a, b]$. As in the case of the tangent bundle, the torsion is a skew-symmetric tensor, i.e. a section of $\bigwedge^{2}\left(E^{*}\right) \otimes E$. The usual construction of the Levi-Civita connection applies, so that, given a field of nondegenerate symmetric inner products on $E$, there is a unique connection without torsion which is compatible with the inner product.

Example 3.4. The flat "Bott connection" on the normal bundle to a foliation is the linearization of the holonomy. But the construction is purely formal and can be extended to the situation where $E^{\prime}$ is any subalgebroid of a Lie algebroid $E$. Namely, we define an $E^{\prime}$-connection on the quotient vector bundle $E / E^{\prime}$ by the rule $\nabla_{a}\langle b\rangle=\langle[a, b]\rangle$, where $a$ and $b$ are sections of $E$ and $\langle\cdot\rangle$ denotes the equivalence class modulo $E^{\prime}$. (We use angled instead of the usual square brackets for the equivalence class to avoid confusion with 
the Lie algebroid operation.) It is straightforward to check that this $\nabla$ is a Lie algebroid representation.

We note that $\nabla$ can be seen as the representation on homology, as in Appendix A of [13], associated to a natural representation up to homotopy of $E$ on the short complex of Lie algebroids $0 \rightarrow E^{\prime} \rightarrow E \rightarrow 0$.

We also introduce a universal enveloping object first defined in a slightly different way by Rinehart [30].

Definition 3.5. Let $(E, \rho,[]$,$) be a complex Lie algebroid on X$, and $\mathcal{T}$ the free associative (i.e. tensor) algebra with generators in $C^{\infty}(X)$ (of degree 0$)$ and $\Gamma(E)$ (of degree 1). The algebra ${ }^{E} \mathrm{Op}$ of E-differential operators on $X$ is defined as $\mathcal{I} / \mathcal{I}$, where $\mathcal{I}$ is the two-sided ideal of $\mathcal{T}$ generated by elements of the form

$$
f \otimes g-f g, \quad f \otimes a-f a, \quad a \otimes b-b \otimes a-[a, b],
$$

and

$$
a \otimes(f b)-(f a) \otimes b-(\rho(a) f) b,
$$

for $a, b \in \mathcal{E}$ and $f, g \in C^{\infty}(X)$.

The grading of $\mathcal{T}$ defines a filtration ${ }^{E} \mathrm{Op}_{n}$ of ${ }^{E} \mathrm{Op}$, and the following result is a straightforward application of Theorem 3.1 in [30].

Lemma 3.6. For any complex Lie algebroid $(E, \rho,[]$,$) over X$, there is a natural isomorphism $\mathrm{Gr}^{E} \mathrm{Op}(X) \simeq \Gamma(X, S(E)$ ), where $S(E)$ is the bundle of symmetric algebras on the fibres of $E$. In particular, the algebra $C^{\infty}(X)$ may be identified with a subalgebra of ${ }^{E} \mathrm{Op}$.

Following Calaque [3], we may also introduce the space of $E$-polydifferential (or multidifferential) operators with its Gerstenhaber bracket operation. (This structure was already suggested by $\mathrm{Xu}[34]$ and used implicitly by Nest and Tsygan [26].)

The usual jet spaces of functions on $X$ are not sensitive enough to the action of $E$, since sections of the isotropy act trivially, so we must use the following generalization.

Definition 3.7. Let $(E, \rho,[]$,$) be a complex Lie algebroid over X$. The space of $E$-jets on $X$ is the linear space ${ }^{E} \mathcal{J}(X)=\operatorname{Hom}_{C^{\infty}(X)}\left({ }^{E} \mathrm{Op}(X), C^{\infty}(X)\right)$.

In the real case, the $E$-jets may be identified with the jets of functions along the units of a (local) groupoid integrating $E$. A similar identification also works in the complex case, though the integration in the sense of [32] may be only formal.

The complex analog of Proposition 2.7 in [26] is:

Proposition 3.8. ${ }^{E} \mathcal{J}(X)$ introduced in Definition 3.7 is the space of global sections of a profinite-dimensional vector bundle ${ }^{E}$ Jets.

We define the "Grothendieck connection" $\nabla^{G}: \mathcal{E} \times{ }^{E} \mathcal{J}(X) \rightarrow{ }^{E} \mathcal{J}(X)$ by

$$
\left(\nabla_{G}(a)(l)\right)(D)=a(l(D))-l(a(D)),
$$

for $l \in \Gamma\left({ }^{E} \mathrm{Jets}\right)\left(={ }^{E} \mathcal{J}(X)\right), a \in \mathcal{E}$ and $D \in{ }^{E} \mathrm{Op}(X)$. As in the real case, this is a flat connection. 


\subsection{A natural complex Lie algebroid on a complex manifold with boundary}

We introduce here the complex Lie algebroid which will be central in what follows. Let $X$ be a complex manifold of dimension $n+1$ with boundary $M$, and let $\mathcal{E}_{M, X}$ be the space of complex vector fields on $X$ (i.e. sections of $T_{\mathbb{C}} X$ ) whose values along $M$ lie in the induced CR structure $G_{M, X}$. Then $\mathcal{E}_{M, X}$ is a module over $C^{\infty}(X)$ and is closed under bracket. The following lemma shows that $\mathcal{E}_{M, X}$ may be identified with the space of sections of a complex Lie algebroid $E_{M, X}$.

Lemma 3.9. $\mathcal{E}_{M, X}$ is a locally free $C^{\infty}(X)$-module.

Proof. Away from the boundary, $\mathcal{E}_{M, X}$ is the same as $T_{\mathbb{C}} M$, hence locally free. Near a boundary point, we may choose a local basis $\bar{v}_{1}, \ldots, \bar{v}_{n}$ of $G_{M, X}$, which we then extend to a linearly independent set of sections of $T^{0,1} X$, still denoted by $\bar{v}_{j}$, defined in an open subset of $X$. We leave the name of the open subset unspecified and will shrink it as necessary. Let $v_{j}$ be the complex conjugate of $\bar{v}_{j}$. These vectors all annihilate $\psi$ on $M$; there is no obstruction to having them annihilate $\psi$ everywhere. Next, we choose a local section $\bar{v}_{0}$ of $T^{0,1} X$ such that $\bar{v}_{0} \cdot \psi=1$, and we let $v_{0}$ be its conjugate. This gives a local basis $(v, \bar{v})$ for the complex vector fields. Such a vector field belongs to $\mathcal{E}_{M, X}$ if and only if, when it is expanded with respect to this basis, the coefficients of $\bar{v}_{0}$ and all the $v_{j}$ vanish along $M$. Since this means that all these coefficients are divisible by $\psi$ with smooth quotient, we get a local basis $\left(u, u^{\prime}\right)$ for $\mathcal{E}_{M, X}$ by setting $u_{0}^{\prime}=\psi \bar{v}_{0}, u_{j}^{\prime}=\bar{v}_{j}$ for $j=1, \ldots, n$, and $u_{j}=\psi v_{j}$ for $j=0, \ldots, n$.

The local basis $\left(u, u^{\prime}\right)$ constructed in the proof above may be thought of as a moving frame, some of whose entries vanish along $M$. The crucial property here is that the structure functions which express Lie brackets in the given frame are smooth up to $M$.

We note that the complex conjugates of the basis vectors are $\bar{u}_{0}=u_{0}^{\prime}$ and $\bar{u}_{j}=$ $\psi u_{j}^{\prime}$ for $j=1, \ldots, n$. The Lie algebroid $E_{M, X}$ does not admit an operation of complex conjugation.

We will also use the coframe $\left(\theta, \theta^{\prime}\right)$ dual to $\left(u, u^{\prime}\right)$. Denoting by $(\gamma, \bar{\gamma})$ the basis of complex-valued 1 -forms dual to $(v, \bar{v})$, we find that $\gamma^{0}=\partial \psi$ and $\bar{\gamma}^{0}=\bar{\partial} \psi$. For the vector bundle $E_{M, X}^{*}$ dual to $E_{M, X}$, we get the local basis of sections $\theta^{j}=(1 / \psi) \gamma^{j}$ for $j=0, \ldots, n$ (so that $\theta^{0}=\partial(\log |\psi|), \theta^{\prime 0}=(1 / \psi) \bar{\gamma}^{0}=(1 / \psi) \bar{\partial} \psi=\bar{\partial}(\log |\psi|)$, and $\theta^{\prime j}=\bar{\gamma}^{j}$ for $j=1, \ldots, n$. The complex conjugates are $\overline{\theta^{0}}=\theta^{\prime 0}$ and $\overline{\theta^{j}}=(1 / \psi) \theta^{\prime j}$ for $j=1, \ldots, n$.

The coframe $\left(\theta, \theta^{\prime}\right)$ is an ordinary coframe on the interior of $X$. Some of these forms blow up along $M$, but the structure functions which express the exterior differentials of these forms in terms of the coframe are smooth up to $M$.

\subsection{Para-Kähler Lie algebroids}

Recall that a pseudo-Kähler structure on a manifold $X$ is a symplectic structure together with a totally complex polarization. This means that we have a (real) nondegenerate 
closed 2-form $\omega$ on $X$ and an integrable subbundle $T^{0,1} X$ of $T_{\mathbb{C}} X$ which is isotropic with respect to the complex extension of $\omega$ and for which $T_{\mathbb{C}} X=\overline{T^{0,1} X} \oplus T^{0,1} X$. We write $T^{1,0} X$ for $\overline{T^{0,1} X}$. The structure is Kähler when the nondegenerate quadratic form $g$ defined on $T^{1,0} X$ by $g(u, v)=\omega(u, J \bar{v})$ is positive definite.

Thinking of a general complex Lie algebroid $E$ over $X$ as a substitute for $T_{\mathbb{C}} X$, it is natural to try to define an analogous notion of Kähler structure, but we lack the operation of complex conjugation. On the other hand, the study of pseudo-Kähler structures often makes little or no use of the quadratic form $g$, but only of the nondegenerate pairing between $T^{1,0} X$ and $T^{0,1} X$ defined by the restriction of the symplectic form. We are thus dealing with a generalization (by complexification and passing from tangent bundles to general Lie algebroids) of the so-called "para-Kähler" [23] or "bilagrangian" [17] structures, which consist of a symplectic form together with a transverse pair of lagrangian foliations.

The following definitions are useful in both the real and complex cases.

Definition 3.10. A [complex] symplectic Lie algebroid is a [complex] Lie algebroid E together with an E-differential 2-form $\omega$ which is ${ }^{E} d$ closed and nondegenerate. A polarization of $(E, \omega)$ is a lagrangian subalgebroid of $E$, i.e. a subbundle which is closed under brackets and maximal isotropic with respect to $\omega$. A [complex] para-Kähler Lie algebroid is a [complex] symplectic Lie algebroid with a splitting $E=E^{1,0} \oplus E^{0,1}$ as the direct sum of two polarizations.

Remark 3.11. The restriction of $\omega$ to $E^{1,0} \times E^{0,1}$ is a nondegenerate pairing which we will continue to denote by $\omega$. This pairing is also the restriction of a unique symmetric inner product on $E$ for which $E^{1,0}$ and $E^{0,1}$ are isotropic.

Example 3.12. The complex Lie algebroid $E_{M, X}$ of Section 3.3 is naturally split as a direct sum $E_{M, X}^{1,0} \oplus E_{M, X}^{0,1}$. Sections of $E_{M, X}^{1,0}$ are fields of holomorphic tangent vectors which vanish on the boundary, while sections of $E_{M, X}^{0,1}$ are fields of antiholomorphic tangent vectors which are tangent to the boundary $\left.\right|^{2}$ Near the boundary, the sections of the two summands are spanned by the $u_{j}$ and $u_{j}^{\prime}$ respectively, for $j=0, \ldots, n$.

In Section 3.6, we will construct a complex symplectic structure for which these summands become lagrangian.

\subsection{The para-Kähler connection}

The complexification of the Levi-Civita connection on a pseudo-Kähler manifold has many nice properties with respect to the splitting of the complexified tangent bundle into its holomorphic and antiholomorphic summands. In fact, it can be constructed directly from this splitting and from the pairing given by the complexified symplectic structure. By imitating this construction, we may construct on any para-Kähler Lie algebroid $E$ a torsion free $E$-connection which is compatible with the para-Kähler structure. (There is

\footnotetext{
2 In the language of [24], we are dealing with a hybrid of the 0 -calculus and the $b$-calculus.
} 
in fact just one connection with these properties.) In the case of a bilagrangian manifold, the construction yields the bilagrangian connection of Hess [17], and in fact, beyond a change of terminology, there is nothing we do here which is not taken from this special case.

Proposition 3.13. Let $\left(E=E^{1,0} \oplus E^{0,1}, \omega\right)$ be a para-Kähler Lie algebroid. There is a unique torsion-free E-connection $\nabla$ on $E$ for which covariant differentiation leaves the para-Kähler structure invariant; i.e. for any $a, b, c \in \Gamma(E), \nabla_{a}$ leaves the splitting invariant, and $\rho(a)(\omega(b, c))=\omega\left(\nabla_{a} b, c\right)+\omega\left(b, \nabla_{a} c\right)$. The curvature of this connection $\nabla$ is in $\left(E^{1,0^{*}} \wedge E^{0,1^{*}}\right) \otimes \operatorname{End}(E)$.

Proof. Our connection $\nabla$ will be built from two flat partial connections on $E$ defined on the summands.

First, identifying $E^{0,1}$ with $E / E^{1,0}$, we have via Example 3.4 an $E^{1,0}$-connection on $E^{0,1}$. Writing $p^{1,0}$ and $p^{0,1}$ for the projection maps associated to the splitting of $E$, we therefore have

$$
\nabla_{a} b^{\prime}=p^{0,1}\left[a, b^{\prime}\right]
$$

for $a \in \Gamma\left(E^{1,0}\right)$ and $b^{\prime} \in \Gamma\left(E^{0,1}\right)$.

This partial connection induces a connection on the dual bundle to $E^{0,1}$, which we identify with $E^{1,0}$ via the pairing $\omega$. The resulting $E^{1,0}$-connection on $E^{1,0}$ is determined by the equation

$$
\omega\left(\nabla_{a} b, c^{\prime}\right)=\rho(a)\left(\omega\left(b, c^{\prime}\right)-\omega\left(b,\left[a, c^{\prime}\right]\right) .\right.
$$

We recall that $\rho$ is the anchor of the Lie algebroid and that we may omit the projection from the last term because $E^{1,0}$ is isotropic for $\omega$.

Putting together these two pieces, we get an $E^{1,0}$-connection $\nabla^{1,0}$ on $E$ which is clearly compatible with the para-Kähler structure.

Now we may interchange the two summands and repeat everything above to get the required $E^{0,1}$-connection $\nabla^{0,1}$ on $E$, and then assemble everything to get the required $E$-connection on $E$. We leave to the reader the exercise of verifying (using the fact that $\omega$ is a closed 2-form) that this connection has zero torsion.

By the Jacobi identity for $E^{1,0}$ and $E^{0,1}$ vector fields, we find that the curvatures of the connections $\nabla^{1,0}$ and $\nabla^{0,1}$ in $\left(\bigwedge^{2}\left(E^{1,0^{*}}\right)+\bigwedge^{2}\left(E^{0,1^{*}}\right)\right) \otimes \operatorname{End}(E)$ vanish. This implies that the curvature $R$ of the $E$-connection $\nabla=\nabla^{1,0}+\nabla^{0,1}$ is a $(1,1)$ form.

We call this connection the para-Kähler connection.

Remark 3.14. A remark made in [12] is still valid here: the para-Kähler connection is the Levi-Civita connection of the symmetric inner product of Remark 3.11. This must be so, since the Levi-Civita connection is unique, and the symmetric inner product, being built in a canonical way from the para-Kähler structure, must be invariant under the paraKähler connection.

Given bases $\xi_{0}, \ldots, \xi_{n}$ and $\xi_{0}^{\prime}, \ldots, \xi_{n}^{\prime}$ of $E^{1,0}$ and $E^{0,1}$ respectively, we will write $\omega_{i j}=\omega\left(\xi_{i}, \xi_{j}^{\prime}\right)$ and $\pi^{i j}$ for the inverse matrix. We may expand the brackets between 
summands as $\left[\xi_{i}, \xi_{j}^{\prime}\right]=m_{i j}^{k} \xi_{k}+m_{i j}^{\prime k} \xi_{k}^{\prime}$. It is straightforward to derive the following formulas for the para-Kähler connection:

$$
\nabla_{\xi_{i}} \xi_{j}=\pi^{l k}\left(\rho\left(\xi_{i}\right) \omega_{j l}-\omega_{j p} m_{i l}^{\prime p}\right) \xi_{k}, \quad \nabla_{\xi_{i}} \xi_{j}^{\prime}=m_{i j}^{\prime k} \xi_{k}^{\prime}
$$

In the usual pseudo-Kähler case, we can choose $\xi_{i}=\partial / \partial z^{i}$ and $\xi_{i}^{\prime}=\partial / \partial \bar{z}^{i}$ to make all the brackets vanish, in which case we get the familiar formulas

$$
\nabla_{\xi_{i}} \xi_{j}=\pi^{l k}\left(\partial \omega_{j l} / \partial z^{i}\right) \xi_{k}, \quad \nabla_{\xi_{i}} \xi_{j}^{\prime}=0
$$

On the other hand, in any para-Kähler Lie algebroid, we may choose the bases $\xi_{0}, \ldots, \xi_{n}$ and $\xi_{0}^{\prime}, \ldots, \xi_{n}^{\prime}$ to be dual to one another with respect to the pairing, so that $\omega_{i j}$ and $\pi^{i j}$ are identity matrices. Then we get

$$
\nabla_{\xi_{i}} \xi_{j}=\sum_{k}-m_{i j}^{\prime k} \xi_{k}, \quad \nabla_{\xi_{i}} \xi_{j}^{\prime}=\sum_{k} m_{i j}^{\prime k} \xi_{k}^{\prime}
$$

As a result of the above observation, we see that all the calculations of Kähler geometry can be carried out in an arbitrary para-Kähler Lie algebroid. This has the following consequence.

Corollary 3.15. Let $X$ be a manifold (possibly with boundary), and $E \rightarrow X$ a paraKähler Lie algebroid whose anchor $\rho: E \rightarrow T_{\mathbb{C}} X$ is invertible on an open dense subset $\mathcal{U} \subset X$. Suppose that the induced para-Kähler structure on $T_{\mathbb{C}} \mathcal{U}$ comes from a pseudoKähler structure on $\mathcal{U}$. Then all contravariant tensors and multi-differential operators on $\mathcal{U}$ which are constructed from the complex structure, the pseudo-Kähler metric, its curvature and covariant derivatives thereof are the images under $\rho$ of smooth objects defined on all of $E$. In particular, they extend smoothly from $\mathcal{U}$ to $X$.

Remark 3.16. One may apply this corollary to the Berezin transform and all the coefficients in the Berezin and Berezin-Toeplitz products, as analyzed by Engliš. This proves smoothness up to the boundary of these constructions without any extra work. Also, we can get smoothness of the canonical form (see Proposition 4.7), since it is the Ricci form of the canonical connection. However, to identify the Berezin-Toeplitz product with an $E$-product in Theorem 4.9 , we need the machinery of formal integrals used by Karabegov and Schlichenmaier [20].

Remark 3.17. It is a much more delicate problem to decide, when the pseudo-Kähler structure on $\mathcal{U}$ is positive definite, whether elliptic analysis can be used as in the compact Kähler setting to get results valid on all of $X$. For instance, under what conditions on the singularities of $\rho$ does the deformation quantization with separation of variables, which extends smoothly to $X$, arise from a Berezin-Toeplitz symbol calculus as is the case in Theorem 4.9 below? 


\subsection{The para-Kähler Lie algebroid near a pseudoconvex boundary}

We return to our study of the Poisson geometry of a complex manifold $X$ with pseudoconvex boundary $X$, now using the complex Lie algebroid $E_{M, X}$. We begin by showing that the symplectic structure $\sigma_{-} \log \psi$, which is singular along $M$, is perfectly regular as an $E_{M, X}$-symplectic structure.

Theorem 3.18. The pullback of $\sigma_{-\log \psi}$ to $E_{M, X}$ is a smooth section of $\bigwedge^{2} E_{M, X}^{*}$. This section is nondegenerate along $M$, hence on a neighborhood of $M$, if and only if $M$ is Levi nondegenerate.

Proof. We will express $\sigma_{-\log \psi}$ in terms of our bases of sections of $T_{\mathbb{C}}^{*} X$ and $E_{M, X}^{*}$. First of all, with sums over repeated indices ranging from 1 to $n$, we have

$$
\partial \bar{\partial} \psi=a \gamma^{0} \wedge \bar{\gamma}^{0}+b_{k} \gamma^{0} \wedge \bar{\gamma}^{k}+\bar{b}_{k} \gamma^{k} \wedge \bar{\gamma}^{0}+c_{j k} \gamma^{j} \wedge \bar{\gamma}^{k},
$$

where $a$ is real and the matrix $c_{j k}$ is hermitian; it is the matrix of the Levi form. From this we get

$$
\begin{aligned}
(1 / i) \sigma_{-\log \psi}= & (1 / \psi)\left(a \gamma^{0} \wedge \bar{\gamma}^{0}+b_{k} \gamma^{0} \wedge \bar{\gamma}^{k}+\bar{b}_{k} \gamma^{k} \wedge \bar{\gamma}^{0}+c_{j k} \gamma^{j} \wedge \bar{\gamma}^{k}\right) \\
& +\left(1 / \psi^{2}\right) \gamma^{0} \wedge \bar{\gamma}^{0} \\
= & -(1 / \psi)\left(a \psi \theta^{0} \wedge \psi \theta^{\prime 0}+b_{k} \psi \theta^{0} \wedge \theta^{\prime k}+\bar{b}_{k} \psi \theta^{k} \wedge \psi \theta^{\prime 0}+c_{j k} \psi \theta^{j} \wedge \theta^{\prime k}\right) \\
& +\left(1 / \psi^{2}\right) \psi \theta^{0} \wedge \psi \theta^{\prime 0} \\
= & (1-\psi a) \theta^{0} \wedge \theta^{\prime 0}-b_{k} \theta^{0} \wedge \theta^{\prime k}-\bar{b}_{k} \psi \theta^{k} \wedge \theta^{\prime 0}-c_{j k} \theta^{j} \wedge \theta^{\prime k} .
\end{aligned}
$$

Along $M$, where $\psi=0$, this becomes

$$
\theta^{0} \wedge\left(\theta^{\prime 0}-b_{k} \theta^{\prime k}\right)-c_{j k} \theta^{j} \wedge \theta^{\prime k}
$$

This is clearly smooth as a section of $\bigwedge^{2} E_{M, X}^{*}$, and its nondegeneracy is equivalent to that of the matrix $c_{j k}$, i.e. to that of the Levi form.

We get another proof of the smooth extension theorem obtained earlier from normal form theory.

Corollary 3.19. The Poisson structure $\pi_{-\log \psi}$ obtained by inverting $\sigma_{-\log \psi}$ near $M$ extends smoothly over $M$.

Proof. On the complement of $M$, near $M$, this Poisson structure is the pushforward of the section of $\bigwedge^{2} E_{M, X}$ obtained by inverting the pullback of $\omega_{-} \log \psi$. We have just seen that the inverse of this pullback extends smoothly over $M$, hence so does its pushforward.

We next turn to the hermitian hessian itself, related to the 2-form $\sigma_{-} \log \psi$ by the formula

$$
g_{-\log \psi}(x, y)=\sigma_{-\log \psi}(x, J y) .
$$

The following result is part of a classical lemma usually attributed to Oka and Lelong. 
Proposition 3.20. If $\psi$ is any defining function for the strongly pseudoconvex boundary $M$ of $X$, then $-\log \psi$ is strongly plurisubharmonic (i.e. $g_{-} \log \psi$ is positive definite) on the complement of $M$ in some neighborhood of $M$ in $X$.

Proof. The quadratic form $q(x)=g_{-} \log \psi(x, \bar{x})$ is expressed in terms of the components of $x$ in our special basis by

$$
\begin{aligned}
q(x)= & -(1 / \psi)\left(a \gamma^{0}(x) \bar{\gamma}^{0}(x)-b_{k} \gamma^{0}(x) \bar{\gamma}^{k}(x)-\bar{b}_{k} \gamma^{k}(x) \bar{\gamma}^{0}(x)-c_{j k} \gamma^{j}(x) \bar{\gamma}^{k}(x)\right) \\
& +\left(1 / \psi^{2}\right) \gamma^{0}(x) \bar{\gamma}^{0}(x) .
\end{aligned}
$$

The corresponding hermitian matrix is the positive function $1 / \psi^{2}$ times the $(1+n) \times$ $(1+n)$ block matrix with $1-\psi a$ in the upper left hand corner, $-\psi c_{j k}$ in the lower right block, and $-\psi b_{k}$ and its adjoint in the off-diagonal row and column. Since $c_{j k}$ is the matrix of the Levi form, it is negative definite when $M$ is strongly pseudoconvex. By the Sylvester criterion (hermitian version), the entire matrix will be positive definite if its determinant is positive. Expanding this determinant (if we ignore the overall factor $1 / \psi^{2}$ ) in minors of the top row, we obtain $\psi^{n}$ times the determinant of $-c_{j k}$ plus terms divisible by $\psi^{n+1}$. Sufficiently close to the boundary, the sum must be positive.

Proposition 3.21. If (with notation as above) the function - $\log \psi$ is strictly plurisubharmonic throughout the interior of $X$, then the Kähler metric $g_{-} \log \psi$ on the interior of $X$ extends to a para-Kähler Lie algebroid structure on $E_{M, X}$.

Proof. We have only to show that the summands in the splitting of Example 3.12 are lagrangian with respect to the symplectic form. But this follows immediately by continuity from the corresponding fact on the interior.

The nondegenerate pairing $\omega: E_{M, X}^{1,0} \times E_{M, X}^{0,1} \rightarrow \mathbb{C}$ is given in our basis of sections by

$$
\beta=(1-\psi a) \theta^{0} \otimes \theta^{\prime 0}-b_{k} \theta^{0} \otimes \theta^{\prime k}+\bar{b}_{k} \psi \theta^{k} \otimes \theta^{\prime 0}-c_{j k} \theta^{j} \otimes \theta^{\prime k}
$$

\section{Quantization}

Deformation quantization on closed Kähler manifolds can be accomplished as a byproduct of Berezin-Toeplitz quantization. See, for instance, [2] [16] and references therein 3 On the other hand, Karabegov [19] studied special formal deformation quantization adapted to the Kähler structure, and he and Schlichenmaier [20] linked the two approaches.

In this section, we will show how to extend the work cited above to the case of Kähler manifolds with pseudoconvex boundary, using para-Kähler Lie algebroids.

\footnotetext{
3 This work applies only to the case where the symplectic structure is integral, but Melrose [25] has shown how to extend the method to the nonintegral case.
} 


\subsection{Definitions}

We start with the "complexification" of a basic definition of Nest and Tsygan [26] (suggested already by Xu [34]).

Definition 4.1. Let $(E, \omega)$ be a complex symplectic Lie algebroid over $X$. An E-star product on $X$ is a formal series of $E$-bidifferential operators

$$
B=1 \otimes 1+\sum_{k \geq 1}(i \hbar)^{k} B_{k}, \quad B_{k} \in{ }^{E} \mathrm{Op}(X) \otimes{ }^{E} \mathrm{Op}(X),
$$

which is associative in the sense that the Gerstenhaber bracket $[B, B]$ is equal to zero, and for which the antisymmetrization of $B_{1}$ is the E-bivector field $\pi$ inverse to $\omega$.

Pushing $B$ forward by the anchor of $E$, we obtain a formal series of $T_{\mathbb{C}} X$-bidifferential operators which gives a star product $\star$ for the Poisson structure which is the pushforward of $\pi$.

Now we extend to the para-Kähler case the notion of quantization with separation of variables.

Definition 4.2. If $E$ is a para-Kähler Lie algebroid, we call an E-star product bipolarized if the bidifferential operators $B_{k}$ all belong to ${ }^{E^{1,0}} \mathrm{Op}(X) \otimes{ }^{E^{0,1}} \mathrm{Op}(X)$.

A bipolarized $E$-star product has the property that $f \star g=f g$ whenever $f$ is an antiholomorphic function or $g$ is holomorphic. When the anchor of $E$ is injective, even on a dense subset of $X$, this property implies that the star product is bipolarized. In the Kähler case, this means that, after changing the sign of the complex structure (or replacing the product by its opposite), we are dealing with a star product with separation of variables in the sense of [19].

\subsection{Bipolarized star products}

Nest and Tsygan [26] showed that the quantization method of Fedosov [14] extends immediately to (real) symplectic Lie algebroids to produce "Weyl-type" star products. Their extension works for complex Lie algebroids as well. On the other hand, Neumaier [27] showed that, when one starts the Fedosov construction with a "bipolarized" symplectic connection (such as the Levi-Civita connection for a pseudo-Kähler manifold) and an "(anti)Wick-type" bipolarized product on the tangent spaces, the resulting star product is bipolarized. In this section, we combine the two constructions above to obtain bipolarized $E$-star products on para-Kähler Lie algebroids.

The idea of the construction of the $E$-star products can be summarized as follows.

In this paragraph, we suppose that our para-Kähler Lie algebroid can be integrated in some sense to an $s$-connected groupoid $G \rightrightarrows X$. The sections of our Lie algebroid can be viewed as left $G$-invariant vector fields along the $s$-fibers of $G$. The symplectic Lie algebroid structure defines a $G$-invariant para-Kähler structure on each $s$-fiber. Therefore, each $s$-fiber of $G$ becomes a para-Kähler manifold and in particular a symplectic 
manifold. $G$ is canonically foliated by the $s$-fibers with equal dimensions, and therefore becomes a regular Poisson manifold, and $X$ is a complete transversal to this foliation. A para-Kähler connection on $X$ can be lifted to a $G$-invariant symplectic connection on $G$. Given a "symplectic connection" on a regular Poisson manifold, we can use Fedosov's construction (Neumaier's construction in [27]) to obtain a star product on $G$. Since the para-Kähler form and the para-Kähler connection are both $G$-invariant, this product we obtain on $G$ is also $G$-invariant, and therefore can be expressed by a $G$-invariant bidifferential operator on $G$, which is a Lie algebroid bidifferential operator and actually bipolarized if the characteristic form is bipolarized.

The construction defined above is based on the uncertain notion of integration of a complex Lie algebroid. We can bypass this problem by working with the $E$-jets introduced in Definition 3.7, which can be viewed as the infinite jets along $X$ of smooth functions on $G$. The Grothendieck connection defines a natural lift of the Lie algebroid action to the infinite jets. Therefore, we can construct a bipolarized star product by working with $E$-jets.

We begin our construction with the fiberwise anti-Wick product. Let $E$ be a paraKähler Lie algebroid over $X$. Then each fiber $E_{x}$ has a natural translation-invariant paraKähler structure given by the symplectic form and the lagrangian subspaces $E_{x}^{1,0}$ and $E_{x}^{0,1}$. Given a basis $\xi_{0}, \ldots, \xi_{n} \in E^{1,0}, \xi_{0}^{\prime}, \ldots, \xi_{n}^{\prime} \in E^{0,1}$ and the dual basis $\theta^{0}, \ldots, \theta^{n} \in$ $E^{0,1^{*}}, \theta^{\prime 0}, \ldots, \theta^{\prime n} \in E^{1,0^{*}}, \omega$ is expressed as $\omega_{i j} \theta^{i} \wedge \theta^{\prime j}$.

We define a bipolarized star product on the algebra

$$
W_{x}:=\mathbb{C}\left[\eta^{0}, \ldots, \eta^{n}, \eta^{\prime 0}, \ldots, \eta^{\prime n}\right][[\hbar]]
$$

of $\mathbb{C}[[\hbar]]$-valued polynomial functions on $E_{x}$ by

$$
f * g:=\exp \left(-\frac{i \hbar}{2} \pi^{i j} \frac{\partial}{\partial \eta^{\prime i}} \otimes \frac{\partial}{\partial \eta^{j}}\right)(f \otimes g),
$$

where $\left(\pi^{i j}\right)$ is the inverse matrix to $\left(\omega_{i j}\right)$. Taking a union of the algebras $W_{x}, x \in X$, we obtain a formal anti-Wick algebra bundle $\mathcal{W}$.

In the following, we adapt Fedosov's construction of star products on symplectic manifolds to our situation.

The para-Kähler connection introduced in Section 3.5 naturally lifts to a connection denoted $\nabla^{l c}$ on the anti-Wick algebra bundle $\mathcal{W}$.

Definition 4.3. A Fedosov connection on $\mathcal{W}$ is a flat connection $D$ on $\mathcal{W}$ of the form $D=$ $\nabla^{l c}+A$, with $A \in \Omega^{1}(M, \operatorname{End}(\mathcal{W}))$ and $D^{2}(a)=(i / \hbar)[\Omega, a]=0$ for all $a \in \Gamma(\mathcal{W})$. $\Omega$ is a $\mathbb{C}[[\hbar]]$-valued 2-form and is usually called the Weyl curvature of the connection $D$.

The following theorem is an extension of Fedosov's theorem on symplectic manifolds to para-Kähler manifolds.

Theorem 4.4. Let $\mu$ be an element of $-\omega+\hbar^{E^{1,0}} \Omega^{1}(X, \mathbb{C}) \wedge E^{0,1} \Omega^{1}(X, \mathbb{C})[[\hbar]]$ such that $d \mu=0$. There exists an $\operatorname{End}(\mathcal{W})$-valued $E$-form $A_{\mu}$ on $X$ such that $\nabla_{\mu}=\nabla^{l c}+$ $A_{\mu}$ defines a Fedosov connection on $\mathcal{W}$ with $\nabla_{\mu} A_{\mu}+\frac{1}{2}\left[A_{\mu}, A_{\mu}\right]=\mu$. The complexes $\left({ }^{E} \Omega(X, \mathcal{W}), \nabla_{\mu}\right)$ and $\left({ }^{E} \Omega\left(X,{ }^{E}\right.\right.$ Jets $\left.\left.\otimes \mathcal{W}\right), \nabla_{G}+\nabla_{\mu}\right)$ are acyclic in positive dimensions. 
Proof. The proof of this theorem is an application of Fedosov's iteration method. The following construction is a generalization of Theorem 3.1 in [27]. We outline the main steps in the following, and omit the detail check.

Notice that the anti-Wick algebra is naturally graded by the sum of the degree of the polynomial and the power of $\hbar$. Therefore, the algebra ${ }^{E} \Omega(X, \mathcal{W})$ is graded by the total degree, which is denoted by "deg". Following Fedosov [14], we introduce operations $\delta$ and $\delta^{-1}$ on ${ }^{E} \Omega(X, \mathcal{W})$, as follows:

$$
\begin{aligned}
\delta(a) & =\sum_{i}\left(\theta^{i} \wedge \frac{\partial}{\partial \eta^{i}} a+\theta^{\prime i} \wedge \frac{\partial}{\partial \eta^{\prime i}} a\right), \\
\delta^{-1}(a) & =\frac{1}{\operatorname{deg}(a)} \sum_{i}\left(\eta^{i} \cdot \iota_{\theta^{i}} a+{\eta^{\prime}}^{i} \cdot \iota_{\theta^{\prime}} a\right),
\end{aligned}
$$

for homogeneous $a \in{ }^{E} \Omega(X, \mathcal{W})$ with positive degree. We look for $A_{\mu}$ of the form $-\delta+(i / \hbar)\left[r_{\mu}, \cdot\right]$, where $r_{\mu}$ is in ${ }^{E} \Omega^{1}(X, \mathcal{W})$ with total degree 5 .

According to $\nabla_{\mu} A_{\mu}+\frac{1}{2}\left[A_{\mu}, A_{\mu}\right]=\mu, r_{\mu}$ is the unique solution of the equations

$$
\delta r_{\mu}=-\omega+\nabla^{l c} r_{\mu}+\frac{i}{\hbar} r_{\mu} * r_{\mu}+R^{l c}-\mu, \quad \delta^{-1}\left(r_{\mu}\right)=0,
$$

where $R^{l c}$ is the curvature of $\nabla^{l c}$, which is in $E^{1,0^{*}} \wedge E^{0,1^{*}}$.

The solution of equation (5) can be obtained by iteration, using the following relation:

$$
r_{\mu}=\delta^{-1}\left(r_{0}\right)+\delta^{-1}\left(\nabla^{l c} r_{\mu}+\frac{i}{\hbar} r_{\mu} * r_{\mu}\right),
$$

where $r_{0}=-\omega+R^{l c}-\mu$.

By the flat connection $\nabla_{\mu}$ constructed in Theorem 4.4 we have isomorphisms

$$
\tau: C^{\infty}(X)[[\hbar]] \rightarrow \operatorname{ker}\left(\left.\nabla_{\mu}\right|_{E} \Omega^{0}(X, \mathcal{W})\right.
$$

and

$$
{ }^{E} \mathcal{J}(X) \rightarrow \operatorname{ker}\left(\left.\left(\nabla_{G}+\nabla_{\mu}\right)\right|_{E} \Omega^{0}\left(X,{ }^{E} \operatorname{Jets} \otimes^{E} \mathcal{W}\right)\right) .
$$

By the isomorphism $\sqrt{7}$, the fiberwise product on ${ }^{E} \Omega^{0}\left(X,{ }^{E}\right.$ Jets $\left.\otimes{ }^{E} \mathcal{W}\right)$ defines an associative product $*$ on the space of $E$-jets. By the duality between the space of $E$ jets and ${ }^{E} \mathrm{Op}(X)$, the product $*$ defines a left $C^{\infty}(X)$-module map $\chi:{ }^{E} \mathrm{Op}(X) \rightarrow$ ${ }^{E} \mathrm{Op}(X) \otimes_{C^{\infty}(X)}{ }^{E} \mathrm{Op}(X)$, i.e. $l_{1} * l_{2}(D)=l_{1} \otimes l_{2}(\chi(D))$. In particular, evaluating $\chi$ on the constant function 1 in ${ }^{E} \mathrm{Op}(X)$, we obtain an $E$-bidifferential operator $\chi(1)$.

By arguments like those used for Theorem 4.3 of [26], we find that $\chi(1) \in{ }^{E} \mathrm{Op}(X) \otimes$ ${ }^{E} \mathrm{Op}(X)$ defines an associative $E$-star product.

Lemma 4.5. For any $E$-jet $f$ constant along $E^{1,0}$ in an open set $U$ of $X,\left.\chi(1)(f, g)\right|_{U}=$ $\left.f * g\right|_{U}=\left.f g\right|_{U}$ for all $g \in{ }^{E} \mathcal{J}(X)$.

For any $E$-jet $g$ constant along $E^{0,1}$ in an open set $V$ of $X,\left.\chi(1)(f, g)\right|_{V}=\left.f * g\right|_{V}=$ $\left.f g\right|_{V}$ for all $f \in{ }^{E} \mathcal{J}(X)$. 
Proof. When $E^{1,0}$ and $E^{0,1}$ are the holomorphic and antiholomorphic tangent bundles of a Kähler manifold, Lemma 4.5] is Proposition 4.4 of [27]. The present case is a small generalization thereof. In the following, we sketch the proof.

We study the isomorphism $\sqrt{6}$ in more detail. Let $\hat{f}$ denote the lifting of an $E$-jet $f$ to a flat section in ${ }^{E} \Omega^{0}\left(X,{ }^{E}\right.$ Jets $\left.\otimes{ }^{E} \mathcal{W}\right)$. Then $\hat{f}$ is the only solution of the differential equation $\nabla_{\mu} \hat{f}=0$ with $\left.\hat{f}\right|_{\eta^{i}=\eta^{i}=0}=f$. We write $\hat{f}=\sum_{k} \hat{f}^{(k)}$ as a sum of its homogeneous degree $k$ components. $\hat{f}^{(k)}$ can be constructed through the following iteration:

$$
\begin{aligned}
\hat{f}^{0} & =f \\
\hat{f}^{(p)} & =\delta^{-1}\left(\nabla^{l c} \hat{f}^{(p)}+\frac{i}{\hbar} \sum_{k=0}^{p-1}\left[r_{\mu}^{(k+2)}, \hat{f}^{(p-k)}\right]\right),
\end{aligned}
$$

where $r_{\mu}^{(k+2)}$ is the degree $k+2$ component of $r_{\mu}$.

The product $*$ on ${ }^{E} \mathcal{J}(X)$ is then defined to be $f * g:=\sigma(\hat{f} \circ \hat{g})$, where $\sigma$ : ${ }^{E} \Omega^{0}\left(X,{ }^{E}\right.$ Jets $\left.\otimes{ }^{E} \mathcal{W}\right) \rightarrow \Omega^{*}\left(X,{ }^{E}\right.$ Jets $)$ by setting all $\eta^{i}, \eta^{\prime}$ equal to 0 and $\circ$ is the fiberwise multiplication in ${ }^{E} \Omega^{0}\left(X,{ }^{E}\right.$ Jets $\left.\otimes{ }^{E} \mathcal{W}\right)$.

To prove Lemma 4.5, we look at each fiber $W$ of the bundle $\mathcal{W}$. In (4), we notice that the product $*$ in the first component only involves $\eta^{\prime}$, and in the second component only involves $\eta$. Due to this property of $*$, we introduce on $W$ a projection $\tau^{1,0}: W \rightarrow W$ onto the component of $\mathbb{C}\left(\eta^{\prime 0}, \ldots, \eta^{\prime n}\right)[[\hbar]]$, the $(1,0)$ component, and a projection $\tau^{0,1}$ : $W \rightarrow W$ onto the component of $\mathbb{C}\left(\eta^{0}, \ldots, \eta^{n}\right)[[\hbar]]$, the $(0,1)$ component. $\tau^{1,0}$ and $\tau^{0,1}$ lift onto $\mathcal{W}$ naturally; denote the composition of $\tau^{1,0}$ and $\hat{f}$ by $\hat{f}^{1,0}$ and similarly the composition of $\tau^{0,1}$ and $\hat{g}$ by $\hat{g}^{0,1}$.

We observe that $f * g$ can be rewritten as $\sigma\left(\tau^{1,0}(\hat{f}) \circ \tau^{0,1}(\hat{g})\right)$, and therefore it is enough to construct $\hat{f}^{1,0}$ and $\hat{g}^{0,1}$ to compute $f * g$. Following the idea of Proposition 4.2 of [27], we can restrict to $E^{1,0}$ and $E^{0,1}$ to calculate $\hat{f}^{1,0}$ and $\hat{g}^{0,1}$. They can be constructed by the following iterations analogous to 8 :

$$
\begin{aligned}
& \hat{f}^{1,0}=f+\delta_{1,0}^{-1}\left(\nabla_{1,0}^{l c} \hat{f}^{1,0}+\frac{1}{\hbar} \tau^{1,0}\left(\hat{f}^{1,0} \circ r_{\mu}^{1,0}\right)\right), \\
& \hat{g}^{0,1}=g+\delta_{0,1}^{-1}\left(\nabla_{0,1}^{l c} \hat{g}^{0,1}+\frac{1}{\hbar} \tau^{0,1}\left(\hat{g}^{0,1} \circ r_{\mu}^{0,1}\right)\right),
\end{aligned}
$$

where $\nabla_{1,0}^{l c}, \nabla_{0,1}^{l c}$, and $r_{\mu}^{1,0}, r_{\mu}^{0,1}$ are the restrictions of $\nabla$ and $r_{\mu}$ to their components in $E^{1,0}$ and $E^{0,1}$.

With the formulas above, it is straightforward to check that if $f$ is constant along $E^{1,0}$, then $\hat{f}^{1,0}=f$ and $f \star g=f g$, and the same holds for the $E^{0,1}$-component.

Proposition 4.6. The isomorphisms (6) and (7) define a bipolarized E-deformation of a para-Kähler Lie algebroid.

Proof. We need to show that $\chi(1) \in{ }^{E^{1,0}} \mathrm{Op}(X) \otimes E^{0,1} \mathrm{Op}(X)$, but this is implied by Lemma 4.5 


\subsection{Strictly pseudoconvex boundary of a complex manifold}

Proposition 4.6 shows that the para-Kähler Lie algebroid associated to the boundary of a complex domain has a polarized $E$-star product. On the other hand, in [8], Engliš constructed a differential star product on a bounded pseudoconvex domain by BerezinToeplitz quantization. In this subsection, we identify Engliš' star product with what we constructed in Proposition 4.6.

Engliš' Berezin-Toeplitz quantization is an example of a deformation quantization with separation of variables. In Theorem 5.9 of [20], Karabegov and Schlichenmaier identified the opposite of the Berezin-Toeplitz star product with a star product with separation of variables whose Karabegov form is equal to $-(1 / i \hbar) \omega+\omega_{\text {can }}$ and characteristic form is $(1 / i \hbar) \omega-\omega_{\text {can }} / 2 i$, where $\omega_{\text {can }}$ is the curvature form of the canonical line bundle.

Proposition 4.7. Near the boundary $M$ of a pseudoconvex domain $X$, the canonical form $\omega_{\text {can }}$ pulls back to a smooth section of $E_{M, X}^{1,0^{*}} \otimes E_{M, X}^{0,1^{*}}$.

Proof. According to the definition given in [20], $\omega_{\text {can }}$ is equal to $-i \partial \bar{\partial} v$, where $i^{n+1} e^{v} d z d \bar{z}$ is the symplectic volume. (We write $d z$ for $d z^{1} \cdots d z^{n+1}$.) To calculate $\omega_{\text {can }}$, we follow the calculation in Proposition 2.6. where the volume form is shown to be

$$
\left(-\frac{\partial \bar{\partial} \psi}{\psi}+\frac{\partial \psi \wedge \bar{\partial} \psi}{\psi^{2}}\right)^{n+1}=\left(-\frac{\partial \bar{\partial} \psi}{\psi}\right)^{n+1}+\left(-\frac{\partial \bar{\partial} \psi}{\psi}\right)^{n} \wedge \frac{\partial \psi \wedge \bar{\partial} \psi}{\psi^{2}}
$$

This form can be written as

$$
\psi^{-n-2} \frac{\operatorname{det}\left(\psi \partial_{i} \bar{\partial}_{j} \psi-\partial_{i} \psi \bar{\partial}_{j} \psi\right)}{\psi^{n}} d z d \bar{z}
$$

It has been shown in Theorem 3.18 that $\nu_{0}=\operatorname{det}\left(\psi \partial_{i} \bar{\partial}_{j} \psi-\partial_{i} \psi \bar{\partial}_{j} \psi\right) / \psi^{n}$ is nonzero and smooth up to the boundary. Therefore, $v$ is equal to $\log \left(\psi^{-n-2} v_{0}\right)=-(n+2) \log (\psi)+$ $\log \left(v_{0}\right)$, and $\partial \bar{\partial} v$ is

$$
-(n+2) \partial \bar{\partial} \log (\psi)+\partial \bar{\partial}\left(\log \left(v_{0}\right)\right) .
$$

To show that $\partial \bar{\partial} \nu$ pulls back to a smooth section of $E_{M, X}^{1,0^{*}} \wedge E_{M, X}^{0,1^{*}}$, we may check the two terms separately.

1. $-(n+2) \partial \bar{\partial} \log (\psi)$ : By Theorem 3.18 д $\partial \bar{\partial} \log (\psi)$ pulls back to be a smooth section of $E_{M, X}^{1,0^{*}} \wedge E_{M, X}^{0,1^{*}}$.

2. $\partial \bar{\partial}\left(\log \left(v_{0}\right)\right)$ : Since $v_{0}$ is nonzero near the boundary, $\partial \bar{\partial} \log \left(v_{0}\right)$ is smooth up to the boundary, and therefore expressed in terms of $\gamma^{i}$ and $\bar{\gamma}^{i}$, which are smooth sections of $\bigwedge^{2} E_{M, X}^{*}$. Finally, the bipolarization of $\partial \bar{\partial}\left(\log \left(v_{0}\right)\right)$ is obvious.

Since $\omega_{\text {can }} \in E^{1,0} \Omega^{1}(X, \mathbb{C}) \wedge E^{0,1} \Omega^{1}(X, \mathbb{C})$, we obtain a bipolarized $E$-star product $\star_{E}$ on $X$ constructed from Proposition 4.6 with the Weyl curvature $\mu$ equal to $\mu=-\omega+i \hbar \omega_{\text {can }}$.

Proposition 4.8. When restricted to the interior of $X$, the Karabegov form of $\star_{E}$ is equal to $-(1 / i \hbar) \omega+\omega_{\text {can }}$. 
Proof. When restricted to the interior of $X, E_{M, X}$ coincides with the tangent bundle of the Kähler manifold $X$. By the locality of our construction of star products, we find that in the interior of $X$, our quantization of a para-Kähler Lie algebroid $E_{M, X}$ coincides with the quantization of the Kähler manifold. This allows us to use Theorem 6.7 and Deduction 6.9 in [27] to obtain the characteristic form.

Now we are ready to state the following theorem.

Theorem 4.9. The opposite Berezin-Toeplitz star product $\star_{B T}^{\prime}$ near a strictly pseudoconvex boundary is equal to the E-star product $\star_{E}$ constructed in Proposition 4.6 and therefore is smooth up to the boundary of $X$.

Remark 4.10. Smoothness up to the boundary for Engliš' Berezin-Toeplitz star product can be directly derived from Corollary 3.15. (See Remark 3.16) But Theorem 4.9 sets up a nice connection between the Berezin-Toeplitz quantization and deformation quantization.

Proof of Theorem 4.9. We know that both the $E$-star product $\star_{E}$ and the opposite Berezin -Toeplitz star product $\star_{B T}^{\prime}$ in the interior of $X$ are star products with separation of variables defined by Karabegov. In Theorem 2 of [19] Karabegov showed that star products with separation of variables on a Kähler manifold $X$ are in one-to-one correspondence to the set of power series in $\hbar$ of closed $(1,1)$ forms on $X$, i.e. $Z_{d R}^{2}(X, \mathbb{C})^{(1,1)}[[\hbar]]$.

We have shown in Proposition 4.8 that the Karabegov form of the $E$-star product ${ }^{\star} E$ is equal to $-(1 / i \hbar) \omega+\omega_{\text {can }}$. A similar calculation as Karabegov and Schlichenmaier did on compact Kähler manifolds showd that the Karabegov form of Engliš' opposite Berezin-Toeplitz star product $\star_{B T}^{\prime}$ is also equal to $-(1 / i \hbar) \omega+\omega_{\text {can }}$. Therefore, we have

$$
\star_{B T}^{\prime}=\star_{E}
$$

And since $\star_{E}$ is smooth up to the boundary of $X, \star_{B T}^{\prime}$ is also smooth up to the boundary, which was already proved in Corollary 3.15

We end the proof by explaining the computation of the Karabegov form of Engliš' opposite Berezin-Toeplitz star product.

Karabegov and Schlichenmaier's calculation in [20] requires the Kähler manifold under consideration to be compact. In [20], they use the compactness assumption to prove that the Berezin-Toeplitz quantization defines a local differential product. Their Propositions 5.1 and 5.2 give an asymptotic expansion of the Berezin transform and the twisted operator product, while Theorem 5.6 gives an asymptotic expansion of the Bergman kernel. All of the remaining calculations are local. For the case of the boundary of a strictly pseudoconvex domain, the analogs of the above asymptotic expansions have been established by Engliš in Theorems 1-3 of [8]. Therefore, we can still use the method of formal integrals as in [20] to compute the Karabegov form of the star product $\star_{B T}^{\prime}$. It turns out to be again equal to $-(1 / i \hbar) \omega+\omega_{\text {can }}$.

Acknowledgments. Research of A. Weinstein was partially supported by NSF Grant DMS-0204100. 


\section{References}

[1] Block, J.: Duality and equivalence of module categories in noncommutative geometry. Preprint math.QA/0509284

[2] Bordemann, M., Meinrenken, E., Schlichenmaier, M.: Toeplitz quantization of Kähler manifolds and $\operatorname{gl}(N), N \rightarrow \infty$ limits. Comm. Math. Phys. 165, 281-296 (1994) Zbl 0813.58026 MR 1301849

[3] Calaque, D.: Formality for Lie algebroids. Comm. Math. Phys. 257, 563-578 (2005) Zbl 1079.53138 MR 2164943

[4] Cannas da Silva, A.: Lectures on Symplectic Geometry. Lecture Notes in Math. 1764, Springer, Berlin (2001) Zbl 1016.53001 MR 1853077

[5] Cannas da Silva, A., Weinstein, A.: Geometric Models for Noncommutative Algebras. Berkeley Math. Lecture Notes, Amer. Math. Soc., Providence (1999) Zbl pre01515267 MR 1747916

[6] Chemla, S.: A duality property for complex Lie algebroids. Math. Z. 232, 367-388 (1999) Zbl 0933.32015 MR 1718638

[7] Eliashberg, Y., Gromov, M.: Convex symplectic manifolds. In: Proc. Sympos. Pure Math. 52, Part 2, Amer. Math. Soc., 135-162 (1991) Zbl 0742.53010 MR 1128541

[8] Engliš, M.: Weighted Bergman kernels and quantization. Comm. Math. Phys. 227, 211-241 (2002) Zbl 1010.32002 MR 1903645

[9] Epstein, C. L.: A relative index on the space of embeddable CR-structures, I, II. Ann. of Math. 147, 1-59, 61-91 (1998) Zbl 0942.32025 $0942.32026 \mid$ MR 1609455 1609451

[10] Epstein, C. L.: Subelliptic $\operatorname{Spin}_{\mathrm{c}}$ Dirac operators, III. The Atiyah-Weinstein conjecture. Preprint math.AP/0507547; Ann. of Math., to appear

[11] Epstein, C. L., Melrose, R. B., Mendoza, G. A.: Resolvent of the Laplacian on strictly pseudoconvex domains. Acta Math. 167, 1-106 (1991) Zbl 0758.32010 MR 1111745

[12] Etayo Gordejuela, F., Santamaría, R.: The canonical connection of a bi-Lagrangian manifold. J. Phys. A 34, 981-987 (2001) Zbl 0990.53081 MR 1820455

[13] Evens, S., Lu, J.-H., Weinstein, A.: Transverse measures, the modular class, and a cohomology pairing for Lie algebroids. Quart. J. Math. 50, 417-436 (1999) Zbl 0968.58014 MR 1726784

[14] Fedosov, B.: A simple geometrical construction of deformation quantization. J. Differential Geom. 40, 213-238 (1994) Zbl 0812.53034 MR 1293654

[15] Gualtieri, M.: Generalized complex geometry. Oxford University DPhil thesis, math.DG/0401221 (2003)

[16] Guillemin, V.: Star products on compact pre-quantizable symplectic manifolds. Lett. Math. Phys. 35, 85-89 (1995) Zbl 0842.58041 MR 1346047

[17] Hess, H.: Connections on symplectic manifolds and geometric quantization. In: Differential Geometrical Methods in Mathematical Physics (Aix-en-Provence/Salamanca, 1979), Lecture Notes in Math. 836, Springer, Berlin, 153-166 (1980) Zbl 0464.58012 MR 0607691

[18] Hitchin, N.: Generalized Calabi-Yau manifolds. Quart. J. Math. 54, 281-308 (2003) Zbl 1076.32019 MR 2013140

[19] Karabegov, A.: Deformation quantizations with separation of variables on a Kähler manifold. Comm. Math. Phys. 180, 745-755 (1996) Zbl 0866.58037 MR 1408526

[20] Karabegov, A., Schlichenmaier, M.: Identification of Berezin-Toeplitz deformation quantization. J. Reine Angew. Math. 540, 49-76 (2001) Zbl 0997.53067

[21] Korányi, A., Reimann, H. M.: Contact transformations as limits of symplectomorphisms. C. R. Acad. Sci. Paris Sér. I Math. 318, 1119-1124 (1994) Zbl 0807.53026 MR 1282355 
[22] LeBrun, C.: Thickenings and conformal gravity. Comm. Math. Phys. 139, 1-43 (1991) Zbl 0733.53056 MR 1116408

[23] Libermann, P.: Sur le problème d'équivalence de certaines structures infinitésimales. Ann. Mat. Pura Apl. 36, 27-120 (1954) Zbl 0056.15401 MR 0066020

[24] Melrose, R. B.: Geometric Scattering Theory. Cambridge Univ. Press, Cambridge (1995) Zbl 0849.58071 MR 1350074

[25] Melrose, R.: Star products and local line bundles. Ann. Inst. Fourier (Grenoble) 54, 15811600 (2004) Zbl 1061.47064 MR 2127859

[26] Nest, R., Tsygan, B.: Deformations of symplectic Lie algebroids, deformations of holomorphic symplectic structures, and index theorems. Asian J. Math. 5, 599-635 (2001) Zbl 1023.53060 MR 1913813

[27] Neumaier, N.: Universality of Fedosov's construction for star products of Wick type on pseudo-Kähler manifolds. Rep. Math. Phys. 52, 43-80 (2003) Zbl 1046.53058 MR 2006726

[28] Nistor, V., Weinstein, A., Xu, P.: Pseudodifferential operators on differential groupoids. Pacific J. Math. 189, 117-152 (1999) Zbl 0940.58014 MR 1687747

[29] Reimann, H. M.: Quasiconformal mappings and pseudoconvex domains. In: XVIth Rolf Nevanlinna Colloquium (Joensuu, 1995), de Gruyter, Berlin, 191-208 (1996). Zbl 0870.32007 MR 1427084

[30] Rinehart, G. S.: Differential forms on general commutative algebras. Trans. Amer. Math. Soc. 108, 195-222 (1963) Zbl 0113.26204 MR 0154906

[31] Weinstein, A.: Some questions about the index of quantized contact transformations. RIMS Kôkyûroku 1014, 1-14 (1997) Zbl 0943.58019 MR 1625440

[32] Weinstein, A.: The integration problem for complex Lie algebroids. In: From Geometry to Quantum Mechanics, Progr. Math. 252, Birkhäuser Boston, Boston MA, 93-109 (2007) Zbl pre05173395 MR 2285039

[33] Weinstein, A., Zambon, M.: Variations on prequantization. In: Travaux Math. XVI, Univ. Luxembourg, 187-219 (2005) Zbl pre05049979 MR 2223158

[34] Xu, P.: Quantum groupoids. Comm. Math. Phys. 216, 539-581 (2001) Zbl 0986.17003 MR 1815717 